\title{
The Dictyostelium Model for Mitochondrial Disease
}

\section{Lisa M. Francione, ${ }^{1}$ Sarah J. Annesley ${ }^{1}$, Sergio Carilla-Latorre ${ }^{2}$, Ricardo Escalante2, Paul R. Fisher ${ }^{*}$}

${ }^{1}$ Department of Microbiology, La Trobe University, VIC 3086, Australia.

${ }^{2}$ Instituto de Investigaciones Biomédicas Alberto Sols, CSIC-UAM, Arturo Duperier 4, 28029-Madrid, Spain.

Key words:

Mitochondrial disease, Dictyostelium, oxidative phosporylation, AMPK, ROS, Complex I, phototaxis, thermotaxis, phagocytosis, pinocytosis, cell growth, Legionella, mitochondrial biogenesis.

* To whom correspondence should be addressed:

Professor Paul Fisher,.

Department of Microbiology,

La Trobe University,

VIC 3086,

Australia.

Tel: + 61394792229

Fax: + 61394791222

Email: P.Fisher@latrobe.edu.au 


\begin{abstract}
Mitochondrial diseases are a diverse family of genetic disorders caused by mutations affecting mitochondrial proteins encoded in either the nuclear or the mitochondrial genome. By impairing mitochondrial oxidative phosphorylation, they compromise cellular energy production and the downstream consequences in humans are a bewilderingly complex array of signs and symptoms that can affect any of the major organ systems in unpredictable combinations. This complexity and unpredictability has limited our understanding of the cytopathological consequences of mitochondrial dysfunction. By contrast, in Dictyostelium the mitochondrial disease phenotypes are consistent, measurable "readouts" of dysregulated intracellular signalling pathways. When the underlying genetic defects would produce coordinate, generalized deficiencies in multiple mitochondrial respiratory complexes, the disease phenotypes are mediated by chronic activation of an energy-sensing protein kinase, AMPK (AMP-activated protein kinase). This chronic AMPK hyperactivity maintains mitochondrial mass and cellular ATP concentrations at normal levels, but chronically impairs growth, cell cycle progression, multicellular development, photosensory and thermosensory signal transduction. It also causes the cells to support greater proliferation of the intracellular bacterial pathogen, Legionella pneumophila. Notably however, phagocytic and macropinocytic nutrient uptake are impervious both to AMPK signalling and to these types of mitochondrial dysfunction. Surprisingly, a Complex I-specific deficiency (midA knockout) not only causes the foregoing AMPK-mediated defects, but also produces a dramatic deficit in endocytic nutrient uptake accompanied by an additional secondary defect in growth. More restricted and specific phenotypic outcomes are produced by knocking out genes for nuclear-encoded mitochondrial proteins that are not required for respiration. The Dictyostelium model for mitochondrial disease has thus revealed consistent patterns of sublethal dysregulation of intracellular signalling pathways that are produced by different types of underlying mitochondrial dysfunction.
\end{abstract}




\section{Introduction}

Mitochondrial diseases are complex degenerative disorders caused by mutations affecting nuclear genes for mitochondrial proteins or mitochondrial genes that encode subunits of the oxidative complexes or translational machinery [1]. In humans, the pathological outcomes include blindness, deafness, epilepsy, heart disease, stroke-like episodes, ataxia, muscle weakness, exercise intolerance, diabetes and kidney disease [2,3]. Mitochondrial dysfunction also plays pathological roles in neurological disorders such as Parkinson's disease and Alzheimer's disease [3-6].

Although much is known about the mtDNA mutations associated with human mitochondrial diseases, the relationship between genotype and phenotype is complicated and poorly understood. To gain further insight into this relationship, $D$. discoideum has been employed as a model organism to study mitochondrial biogenesis and disease [7-11]. The mitochondrial genome of Dictyostelium has been fully sequenced [12], while mitochondrial transcription and RNA processing in this organism have been thoroughly examined [1315]. Mitochondrial disease has been created in Dictyostelium by a variety of methods and the pathological consequences studied. Since Dictyostelium has motile unicellular and multicellular stages with multiple cell types, many phenotypes can be examined including phototaxis, thermotaxis, macropinocytosis, phagocytosis, cell cycle progression and growth, amoeboid motility and chemotaxis, morphogenesis and intracellular growth of pathogens such as Legionella pneumophila. These varied phenotypes represent measurable, reproducible "readouts" of the intracellular signaling pathways that regulate them. By assaying them in mitochondrially diseased Dictyostelium lines, we can gain a better understanding of genotypephenotype relationships in mitochondrial disease, without the overlaid complexities associated with mammalian systems.

To understand the cytopathological pathways involved in mitochondrial diseases it is important to identify and study proteins that play roles in these pathways. One such protein is AMP-activated protein kinase (AMPK), a ubiquitous, highly conserved protein kinase that maintains cellular energy homeostasis in healthy and diseased cells [9,16]. Bokko et al. (2007) [9] and Francione et al. (2009) [11] have shown that signalling by this protein is responsible for diverse cytopathologies seen in Dictyostelium mitochondrial disease. However, Carilla-Latorre et al. (2010) [17] reported recently that, in Complex I-specific disease, additional aberrant signalling pathways become involved so that additional AMPK-independent phenotypes are also observed. This review will highlight the contributions made by the Dictyostelium model to our understanding of mitochondrial biology and disease. The genetic manipulation strategies that have been 
brought to bear and the associated phenotypes in $D$. discoideum are discussed, the role of AMPK is featured and our emerging understanding of Complex I-specific disease is described.

\section{Mitochondrial Biology}

Mitochondria are ubiquitous in eukaryotic cells where they are central to the maintenance of cell function and viability. These organelles, numbering from one to thousands depending on the cell type, are responsible for generating most of the cell's ATP which constitutes the direct or indirect energy source for most cellular functions. ATP is generated by oxidative phosphorylation (OXPHOS) at the inner mitochondrial membrane. The proteins involved in OXPHOS are encoded by genes of both the mitochondrial and nuclear genomes (see Supplementary Table 1). Mutations in these or other genes that affect OXPHOS lead to an array of mitochondrial diseases [18].

\subsection{The mitochondrial genome of humans and D. discoideum}

Each D. discoidem cell contains about 200 copies of the mitochondrial genome which, like that of humans, is a circular molecule. The mitochondrial genome of humans is $16,569 \mathrm{bp}$ [19] whereas in $D$. discoideum it is larger at 55,564 bp [12]. The average coding capacity of the mitochondrial genome in eukaryotes is 40-50 genes. The mitochondrial gene products are mainly required for five processes: respiration and/or OXPHOS, translation and in some organisms also transcription, RNA maturation and protein import $[1,20]$. In mammals, the majority of protein subunits $(>80)$ of the respiratory chain are encoded in the nucleus whereas only 13 subunits are encoded in the mitochondrial genome $[18,20]$. Production of these mitochondrially-encoded proteins requires the import of a variety of nuclear encoded proteins such as the single subunit mitochondrial RNA polymerase, amino acyl tRNA synthetases and the mitochondrial transcription factor A. Molecules required for mitochondrial protein synthesis which are encoded by the human and $D$. discoideum mitochondrial genomes are shown in Supplementary Table 2.

The mitochondrial genes and ORFs in Dictyostelium are all transcribed in the same orientation [12] (Figure 1). Some overlapping of genes is evident in the tightly packed genome, which also exhibits intergenic spacing from several nucleotides to greater than $2 \mathrm{~kb}$. The genome also encodes several ORFs that do not exist in most other organisms and presumably do not play essential universal roles.

All of the genes in the Dictyostelium mitochondrial genome are transcribed to one of eight large polycistronic mRNA transcripts which are further processed to form a variety of smaller monocistronic, dicistronic or tricistronic mature RNA molecules [14]. Recently these eight transcripts were shown to be derived from processing of a single primary RNA transcript $[15,21]$. The transcription initiation site in the 
Dictyostelium mitochondrial genome is located in a noncoding region upstream of $\mathrm{rn} /$ which encodes the large ribosomal subunit RNA (Figure 1) [15].

\subsection{The mitochondrial protein import machinery}

The majority of the $c a .1500$ resident mitochondrial proteins are encoded by nuclear DNA, translated by cytoplasmic ribosomes and imported by the mitochondrial protein import machinery $[20,22]$. These include not only the other metabolic enzymes and "housekeeping" proteins, but also the remaining 70 subunits of the OXPHOS complexes (that assemble with those encoded by mtDNA) as well as factors required for expression and replication of the mitochondrial genome [23]. Defective protein import may lead to mitochondrial respiratory dysfunction, reduced ATP production and mitochondrial disease (eg. Human Deafness Dystonia Syndrome [24]). In turn impairment of respiration can inhibit mitochondrial protein import, since import is energized both directly by the mitochondrial membrane potential $\left(\Delta \psi_{m}\right)$ and by ATP hydrolysis involving proteins such as the mitochondrial heat-shock protein ( $\mathrm{Hsp}) 70$ ( $\mathrm{mtHsp} 70$ ) and mitochondrial import stimulation factor (MSF) $[25,26]$. The mitochondrial protein import machinery also assists in the correct sorting and translocation of proteins to the correct mitochondrial compartments - the matrix, the inner membrane, the intermembrane space and the outer membrane [22].

Although our knowledge of protein import into mitochondria is based mostly on research in S. cerevisiae and N. crassa, similar processes are likely to occur in other species. Tom40, Tom70 and Tom22 were found to be common elements of the mitochondrial protein machinery amongst eukaryotic genome sequences and orthologues are also encoded in the Dictyostelium genome [27].

Interestingly Ahmed et al. (2006) [28] reported that import of some mitochondrial proteins in Dictyostelium occurs cotranslationally in vivo. Cotranslational import of mitochondrial proteins has also been observed in yeast $[29,30]$. Current evidence suggest that it occurs as a result of transport and binding of the mRNA to the mitochondrial surface in a manner that depends on secondary stem-loop structures in the mRNA, often in untranslated regions of the transcript [29].

\section{Human mitochondrial diseases}

Mutations in mitochondrial or nuclear genes that encode components of the translational machinery or the OXPHOS complexes have been associated with human mitochondrial diseases. Due to the presence of mitochondria in all cells of the human body, every tissue can be adversely affected by mutations in the mitochondrial genome. However, there is tissue-specific variation in the numbers of mitochondria, the proportion of mutant mitochondrial genomes, the energy demands of the cell, the isoforms of nuclearencoded mitochondrial proteins that are expressed and the extent of cellular and mitochondrial 
proliferation. Depending on which tissues are affected most in a given individual, this leads to a bewildering complexity in mitochondrial diseases which exhibit a very broad range of possible symptoms

(Supplementary Tables 3 and 4). Individuals with the same mitochondrial disease mutation can exhibit huge variation in clinical symptoms while different mutations can result in similar clinical manifestations. Thus phenotypes associated with human mitochondrial diseases are unpredictable from specific genetic defects.

In mitochondrial diseases, pathogenic mutations in mitochondrial genes usually affect only a subset of the genomes (a state referred to as heteroplasmy) and the extent of the phenotypic outcomes is sometimes related to how large this subset is (the mutant load). This can contribute to a phenomenon whereby a particular pathological outcome is only observed when the mutant load exceeds a particular threshold. A good example of such a threshold is shown by NARP (Neuropathy, Ataxia and Retinitis Pigmentosa) and LS (Leigh Syndrome) which can result from the same mutation. NARP is caused by 70-90\% of mutated mtDNA whereas LS develops if the mutation load is above $90-95 \%$ [31,32]. In some cases, mtDNA mutations must be above a particular threshold for significant ATP depletion and/or symptoms of the disease to occur [33]. Biochemical thresholds that influence the extent to which ATP levels are reduced as a consequence of gene mutations, have been described in detail by Rossignol et al. (2003) [34].

\section{D. discoideum as a model organism for mitochondrial disease}

The genetics, biochemistry and signal transduction processes of $D$. discoideum have been studied extensively and are well understood. With fully sequenced mitochondrial [12] and nuclear [35] genomes, the organism is one of a small number of nonmammalian model organisms recognized by the National Institute of Health (NIH) in the U.S.A. for their importance in biomedical research (http://www.nih.gov/science/models/). Many Dictyostelium genes are orthologues of disease-related genes [35] and this has facilitated the establishment of Dictyostelium models for investigating a variety of human diseases $[10,39,40]$, including mitochondrial disease [21]. In those cases where the relevant experiments have been done, heterologous expression and study of Dictyostelium genes in other organisms, or vice versa, has confirmed directly that the functions of orthologous proteins have been conserved during evolution [10].

\subsection{Life cycle and phenotypes}

What makes Dictyostelium so valuable a model organism is that it combines genetic, biochemical and cell biological tractability with a unique life cycle providing a great variety of readily assayed, reproducible disease phenotypes. The organism grows as isolated amoeboid cells that divide mitotically and obtain nutrients either by phagocytic consumption of bacteria or by macropinocytosis of liquid medium. Starvation induces a developmental programme in which the amoebae differentiate and consequently 
become responsive to an attractant, CAMP, which they now synthesize and secrete. The resulting aggregation process leads to formation of a motile, multicellular organism (the "slug") containing multiple cell types organized in a well-defined spatial pattern. The migratory slug exhibits highly sensitive phototactic and thermotactic responses under control of its specialized anterior cells. After a variable period of migration it forms a fruiting body by a combination of morphogenetic movements and further cell differentiation. This life cycle, with its motile unicellular and multicellular stages, offers diverse phenotypic "readouts" of the signaling pathways that regulate them. These include cell growth and division [38]; amoeboid motility and chemotaxis [39]; phagocytosis and macropinocytosis [40]; phototaxis and thermotaxis [41,42]; cell and tissue differentiation and pattern formation [43]; autophagic cell death [44]; multicellular tissue movement and morphogenesis $[45,46]$. Dictyostelium has also been used to study the interactions between microbial pathogens, such as Legionella pneumophila, and their hosts [47]. The molecular mechanisms of Legionella pathogenesis in $D$. discoideum are similar to those in human cells $[48,49]$. Despite this phenotypic richness, many of the complexities associated with mammalian systems are eliminated in the Dictyostelium model, since all stages of the life cycle can be readily studied in clonally derived, genetically identical cell lines. The study of mitochondrially diseased Dictyostelium strains has accordingly revealed consistent, reproducible outcomes that contrast with the unpredictability of mitochondrial disease in humans.

As mentioned previously human mitochondrial diseases are characterized by pathological or biochemical thresholds. Mitochondrial disease in Dictyostelium has exhibited similar thresholds in that some phenotypes appear more sensitive to mitochondrial dysfunction than others $[7,8,50]$. Although genetic manipulations that would impair respiration resulted in similar phenotypic outcomes, the severity of the phenotypes varied amongst the mitochondrially diseased strains so that particular defects such as slow growth were not observed in every strain $[7-9,50]$. Much of this variation in the severity of the phenotypic aberrations in mitochondrially diseased Dictyostelium was shown to be caused by differences in the severity of the underlying genetic defect. As described in the next section, such differences are readily created and quantitatively measured in Dictyostelium.

\subsection{Genetic manipulation to produce mitochondrial disease in Dictyostelium}

In order to study mitochondrial disease in Dictyostelium various methods have been employed to create sublethal mitochondrial dysfunction. These include $\mathrm{RNA}_{i}$ (interfering RNA) [51] or antisense inhibition of expression of specific mitochondrial proteins [8,9], heteroplasmic disruption of mitochondrial genes $[7,11,52]$ and knockout of nuclear genes encoding nonessential mitochondrial proteins [53-56]. Mitochondrial defects in Dictyostelium have also been generated pharmacologically through the use of ethidium bromide to deplete the cells of mitochondrial DNA [57]. 


\subsubsection{Heteroplasmic targeted disruption}

The first Dictyostelium mitochondrial gene targeted for heteroplasmic disruption was $r n l$, the mitochondrial large ribosomal subunit RNA gene [7]. The original disruption of this gene was a result of a nontargeted insertion into a gene important for phototaxis. Subsequent targeted disruptions confirmed the effect on phototaxis and initiated the study of mitochondrial disease in Dictyostelium [7]. Another Dictyostelium mitochondrial gene rps4, encoding a ribosomal protein, was targeted for heteroplasmic disruption by Inazu et al (1999) [52]. Apart from $\mathrm{rnl}$ and $r p s 4,8$ other mitochondrial genes have been disrupted and regardless of which gene was targeted, the expression of the entire mitochondrial genome was reduced [50]. The strength of signals in Southern blots of the mitochondrial DNA indicates that only a subset of the mitochondrial genomes is affected in these strains i.e. the disruptions are heteroplasmic $[7,50]$ allowing Dictyostelium to be used as a model for human heteroplasmic mitochondrial disease.

\subsubsection{Antisense and RNAi inhibition of nuclear genes encoding essential mitochondrial proteins}

Mitochondrial disease can also be caused by mutations affecting nuclear genes that encode mitochondrial proteins. An example is $h s p A$, which encodes chaperonin 60 (Cpn60), a protein located in the mitochondrial matrix. Cpn60 is required for correctly folding proteins newly imported into the mitochondrial matrix. A deficiency of $\mathrm{Cpn60}$ is linked to a mitochondrial disease in humans, the symptoms of which include severe neurological and developmental defects and depletion of many respiratory enzymes [58-60]. In D. discoideum a Cpn60 insufficiency was created by antisense inhibition of expression of the protein $[8,9]$. Transformation of Dictyostelium with an antisense-inhibition construct produces transformants in each of which a different number of copies of the plasmid are integrated into the nuclear genome. The copy number is stable, easily determined using several techniques $[61,62]$ and correlates with the reduction of expression of the target gene and the phenotypic outcomes $[8,9]$. RNAi constructs have been used similarly to reduce the expression of the catalytic subunit of mitochondrial succinate dehydrogenase (respiratory Complex II) (Lay \& Fisher, unpublished results) and Dd-TRAP1 (D. discoideum Tumor necrosis factor receptor-associated protein), a Hsp90 homologue which translocates to the mitochondria in early differentiation [51]. By analogy with Hsp90 in other organisms, it may play a role in chaperoning some proteins en route to the mitochondria.

\subsubsection{Targeted disruption of nuclear genes encoding nonessential mitochondrial proteins}

While the heteroplasmic disruption of essential mitochondrial genes is possible, it is lethal to disrupt a nuclear gene in Dictyostelium's haploid genome if it encodes an essential mitochondrial protein. Such a gene must supply its encoded protein to every mitochondrion in the cell. However some mitochondrial proteins are not essential for viability. Genes encoding these proteins can be disrupted through 
homologous recombination and the associated phenotypes examined. Such genes include cluA [54], torA (Tortoise) [53], fszA and fszB [55], midA [17,63] and aoxA [64].

\subsection{Dictyostelium mitochondrial disease pathology}

Several broad categories of mitochondrial dysfunction have been studied in the Dictyostelium model (Table 1). The first is generalized oxidative phosphorylation (OXPHOS) defects that are expected to impair multiple respiratory complex deficiencies. Such defects have been studied by sublethal genetic or pharmacological manipulations that reduce but do not eliminate the oxidative phosphorylation (OXPHOS) capacity of the mitochondria. The second category of mitochondrial defects studied in Dictyostelium are those that affect mitochondrial functions other than OXPHOS. Finally, in very recent work, a class of mutant has been found that produces a specific reduction in the activity of only one of the respiratory complexes (Complex I). Each of these categories of mitochondrial dysfunction has contributed to our growing understanding of the cytopathological pathways underlying mitochondrial disease.

\subsubsection{Generalised OXPHOS defects}

The most obvious biochemical consequence of a generalized deficiency in oxidative phosphorylation is a reduced capacity to synthesize ATP. In humans the phenotypic outcomes of mitochondrial disease were thought accordingly to result from a depletion of ATP and the different energy requirements of cellular functions. However, in Dictyostelium it has been shown that mitochondrial disease phenotypes arising from generalized OXPHOS defects (Table 1) do not result simply from a depletion of ATP but from disturbances in intracellular signalling networks. In mitochondrial disease the energy generating capacity of the mitochondria is compromised which results in activation of intracellular energy stress signals. These signals are relayed into various signalling pathways to produce phenotypic affects, some of which are more sensitive to mitochondrial dysfunction than others.

The first mitochondrial disease phenotype to be discovered in Dictyostelium was impaired phototaxis and thermotaxis in the multicellular slug stage of the life cycle [7]. Dictyostelium slugs display extremely sensitive, accurate orientation towards light and in temperature gradients $[47,48]$. Phototaxis and thermotaxis pathways converge early so that almost all of the proteins involved are required for both processes. The photo/thermosensory transduction pathways in Dictyostelium are not completely understood, but many participating molecules have been identified which could be downstream targets of energy stress signalling. These include heterotrimeric $\mathrm{G}$ proteins $[47,48]$; the second messengers $\mathrm{CAMP}$, CGMP, IP 3 and $\mathrm{Ca}^{2+}[47,48]$; signalling proteins such as RasD [65], GefE and GefL [66]; protein kinases such as PKB and ErkB [67]; and cytoskeletal proteins such as GRP125 [68], villidin [69], CAP [70], filamin [71] and FIP [72]. A number of the proteins involved form a photosensory signalling complex that is assembled on 
the scaffolding protein filamin $[67,73]$. The phototaxis and thermotaxis signalling pathways were impaired by mitochondrial disease in all cases tested, regardless of how the mitochondrial dysfunction was caused. Thus heteroplasmic disruption of any of 10 different mitochondrial genes and down regulation of chaperonin 60 all resulted in phototaxis and thermotaxis defects $[7,8,11,50,52]$. Likewise phototaxis was impaired by depletion of mitochondrial DNA using ethidium bromide treatment [57].

The second phenotype to be affected by mitochondrial disease is growth. Dictyostelium cells can grow either on bacterial lawns (obtaining nutrients by phagocytosis) or axenically in liquid medium (obtaining nutrients by macropinocytosis). Mitochondrially diseased Dictyostelium cells showed impaired growth in liquid and on bacterial lawns [7-9]. However these growth defects were not a result of altered phagocytosis or macropinocytosis [9], nor did the cells show alterations to their size, implying a coordinate inhibition of both cell growth and cell cycle progression (Ahmed and Fisher, unpublished data).

The proteins controlling the cell cycle that could be dysregulated in mitochondrial disease include seven cyclins, six cyclin-dependent kinases identified in Dictyostelium and homologues of inhibitors of cell cycle progression such as the retinoblastoma protein $\mathrm{Rb}$, a homologue of which is encoded by rblA [35]. The major signalling pathway controlling cell growth in metazoans involves the protein kinase TOR in a multiprotein complex called TORC1. Inhibition of this pathway involves activation of TSC2 which in turn inactivates Rheb and thereby inactivates TOR $[38,74]$. These proteins are conserved in Dictyostelium [35] but their functional roles in signalling pathways involved in growth control or mitochondrial disease have not been determined. In mammals AMPK inhibits TORC1 directly through the RapTOR subunit [75] and indirectly by activating TSC2 [74]. Because mitochondrial dysfunction compromises ATP generation, AMPK should be chronically activated in mitochondrially diseased cells. This indeed appears to be the case for mitochondrially diseased Dictyostelium cells [9] as well as Parkinson's [76], Alzheimer's [77] and Huntington's [78] disease neurons. As described in greater detail in later sections, chronic AMPK signalling was found to be responsible for the slow growth of mitochondrially diseased Dictyostelium cells [9].

The third phenotype which is consistently affected by mitochondrial dysfunction is the differentiation of cells into stalk and spore cells, with mitochondrially diseased cells showing an increased number of cells directed into the stalk differentiation pathway and mislocalisation of these cells in the multicellular slug. At culmination this results in fruiting bodies with thick short stalks. This effect was seen in mitochondrially diseased cells created either by genetic manipulation $[8,9]$ or by treatment with ethidium bromide [57]. In addition, almost all cells were directed into the stalk differentiation pathway by inhibitors of cyanideresistant respiration that also impaired the mitochondrial membrane potential, loss of which is known to stimulate autophagy [79]. Stalk cell differentiation in Dictyostelium involves a programmed cell death accompanied by the accumulation of autophagic vacuoles. Mitochondrial dysfunction in Dictyostelium thus 
appears to direct cells into an autophagic cell death pathway that may be analogous to the autophagic cell death implicated in neurodegeneration in humans [44].

The last phenotype to be associated with mitochondrial disease in Dictyostelium is altered transition from growth to development and subsequent chemotactic aggregation. Inazu et al (1999) [52] showed that knock down of the mitochondrial rps4 gene resulted in greatly impaired cell aggregation and significantly lower levels of car1, an early developmental gene. Depletion of mitochondrial DNA by ethidium bromide treatment impaired aggregation significantly and reduced the expression of early developmental genes including carA [57]. Aggregation was also impaired in chaperonin 60 or succinate dehydrogenase antisenseinhibited cells resulting in fewer, smaller aggregates [8,9,Lay \& Fisher, unpublished].

\subsubsection{Defects not known to affect OXPHOS}

Mutations affecting the mitochondria have been created which do not damage the energy generating capability of the mitochondria (Table 1). One example is knockout of the mitochondrial fission proteins FszA and FszB [55], whose closest homologues are FtsZ proteins that mediate binary fission in the $\alpha$ proteobacteria, the presumed ancestors of mitochondria. In some eukaryotic lineages such as fungi, animals and plants, no mitochondrial FtsZ proteins have been identified and mitochondrial division is primarily undertaken by FIS1 and DNM1L (dynamin-like protein also known as DRP1) [80]. Mitochondrial fission in Dictyostelium involves both dynamin-like proteins [81] and FtsZ proteins [55], indicating that the latter were retained in the amoebozoan lineage but discarded in some others. Disruption of FszA or FszB impaired mitochondrial division, producing more elongated mitochondria, while disruption of FszB but not FszA also caused growth defects [55]. However, none of the other characteristic mitochondrial disease phenotypes described in the preceding section were observed.

FszB was found localised to submitochondrial bodies of unknown function [55] which are also enriched in another mitochondrial protein TorA (tortoise) [53]. TorA null amoebae move slowly and fail to suppress inappropriate lateral pseudopod formation during chemotaxis, defects which may explain why they grow slowly on bacterial lawns. The specificity of the motility and chemotaxis defects suggests that TorA null cells still produce wild type levels of ATP [53]. There is no human homologue for TorA and it is unknown whether TorA mutant cells display any of the phenotypes typically associated with mitochondrial respiratory dysfunction in Dictyostelium.

A nuclear gene required for normal subcellular localization of mitochondria is cluA. First discovered and functionally characterized in Dictyostelium, cluA has homologues in all eukaryotes whose genomes have been sequenced. CluA null mutants exhibit pronounced perinuclear clustering of the mitochondria in mutant cells [54]. CluA is also involved in cytokinesis since mutant cells were multinucleate and slightly 
impaired in growth [54]. The involvement in cytokinesis is likely to be an indirect result of the contractile ring being physically occluded by large aggregates of mitochondria that block completion of cleavage [82].

Another mitochondrial protein that has been studied genetically is TRAP1, a homologue of mammalian TRAP1 (Tumour Necrosis Factor Receptor-Associated Protein 1). TRAP1 belongs to the Hsp90 molecular chaperone family and localises to the mitochondria as well as to extramitochondrial sites such as the nucleus, secretory granules and cell membranes [83-85]. The protein is localised to the cell cortex of cells growing at low cell densities and translocates to the mitochondria as the cell density of growing cells increases [56]. RNAi inhibition of TRAP1 expression resulted in slow vegetative growth and delayed aggregation, while overexpression resulted in premature aggregation.

The alternative oxidase encoded by aoxA in Dictyostelium is a mitochondrial protein with homologues in animals, plants, fungi and bacteria [86]. It can accept electrons from ubiquinone in place of Complex III and pass them directly to molecular oxygen, thus providing an alternative pathway for electron flow in which protons are pumped across the mitochondrial inner membrane only at Complex I. This process of cyanideresistant respiration appears to serve primarily as an electron sink that prevents leakage of electrons in single electron transfers to oxygen that would form superoxide anions and thence other reactive oxygen species (ROS). Kimura et al. (2010) [64] knocked out aoxA in Dictyostelium and found no obvious phenotypic changes in growth or development under normal conditions. However development in the mutant was completely blocked by millimolar concentrations of $\mathrm{KCN}$ that merely delayed development in the wild type.

\subsubsection{The role of AMPK}

It was first proposed by Wilzcynska et al. (1997) [7] that a cytopathological effect of mitochondrial disease is impairment of signal transduction pathways with some pathways being more sensitive than others. Recently a molecular link between mitochondrial disease and cellular signalling was identified in Dictyostelium in the form of AMP-activated protein kinase (AMPK) [9]. AMPK is an essential sensor and homeostatic regulator of cellular energy status [87]. The serine/threonine protein kinase is a heterotrimer composed of a catalytic $\alpha$ subunit, a regulatory $\gamma$ subunit and a $\beta$ subunit which acts as a scaffold for the other subunits. In mammalian cells there are three genes encoding isoforms of the $\gamma$ subunit and two for each of the $\alpha$ and $\beta$ subunits, whereas in Dictyostelium only one of each subunit is encoded in the genome.

Activation of AMPK occurs when AMP binds to the two Bateman domains on the $\gamma$ subunit relieving its inhibition of the catalytic $\alpha$ subunit. The $\alpha$ subunit is now able to be phosphorylated at a threonine residue by an upstream kinase and is also resistant to dephosphorylation by phosphatases. Because of competition between AMP and ATP for binding to the Bateman domains, AMPK activation is very sensitively regulated 
by AMP/ATP ratios with high ATP levels producing the inactive heterotrimer. Several upstream AMPK kinases have been identified to date - LKB1 [88,89], CaMKK $\alpha$ and $\beta[90,91]$ and TAK1 [92]. Of these, LKB1 is reported to be the major activator [89].

Once activated, AMPK homeostatically rectifies low cellular energy levels by activating pathways that produce ATP while inhibiting others that consume it. These effects are exerted by inhibiting key enzymes in biosynthetic pathways (eg. acyl CoA carboxylase in fatty acid synthesis and 3-hydroxy-3-methylglutaryl-CoA reductase in cholesterol biosynthesis), activating protein translocation (eg. the GLUT4 glucose transporter to the plasma membrane, increasing glucose uptake) [93], and altering gene expression (eg. repression of glucose responsive genes and protein synthesis [94,95], induction of mitochondrial biogenesis $[96,97])$. These actions enable AMPK to regulate the AMP/ATP ratio and maintain healthy energy levels within the cell.

Because of its exquisite sensitivity to AMP/ATP ratios, AMPK exerts its effects prior to a serious depletion in energy. Bokko et al. (2007) [9] provided molecular genetic evidence that AMPK is chronically activated in mitochondrial disease. Mitochondrial disease was created in Dictyostelium by antisense inhibition of chaperonin 60 to produce the defective phenotypes discussed earlier, including defective photo/thermotaxis, growth and morphogenesis. These defective phenotypes were phenocopied by

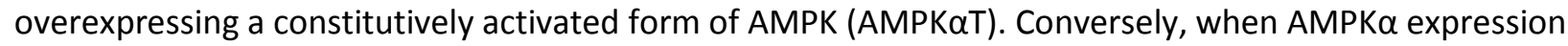
was knocked down in mitochondrially diseased cells (chaperonin 60 antisense inhibition), the defective phenotypes were suppressed. AMPKaT-expressing strains also exhibited increased mitochondrial mass and higher ATP levels, as expected since AMPK stimulates biogenesis and ATP production in mammalian cells. Mitochondrially diseased cells did not show changes in mitochondrial mass or steady state ATP levels presumably due to a balance between reduced biogenesis and ATP production caused by antisense inhibition of chaperonin 60 and increases due to chronic activation of AMPK. However this chronic AMPK hyperactivity in mitochondrially diseased cells leads to permanent dysregulation of the downstream energy-consuming pathways under AMPK's control (Figure 2).

\subsubsection{Dictyostelium and Complex I dysfunction}

NADH:ubiquinone oxidoreductase or Complex I $(\mathrm{Cl})$ is the first and the largest complex of the respiratory chain and couples the oxidation of $\mathrm{NADH}$ to reduction of ubiquinone and the transport of protons. It is formed by 45 protein subunits in mammalian cells that assemble together into a structure of approximately $1 \mathrm{Mda}$ [98]. $\mathrm{Cl}$ has important implications in human pathology due to its role in the generation of reactive oxygen species and the increasing number of diseases whose cause is directly or indirectly associated with $\mathrm{Cl}$ function. About $40 \%$ of inherited disorders of the OXPHOS system involve isolated or combined 
deficiencies in $\mathrm{Cl}$. As described previously these deficiencies present diverse combinations of clinical manifestations, including fatal childhood disorders such as Leigh syndrome. Complex I dysfunction has also been linked to neurodegenerative disorders such as Parkinson's and Alzheimer's diseases [99-101]. Despite its importance in human pathology many aspects of $\mathrm{Cl}$ function, including its regulation, assembly and structure are poorly understood.

$\mathrm{Cl}$ is present in prokaryotes in its minimal version formed by the so-called core subunits. This bacterial complex is able to perform the essential redox reactions required for its function. However eukaryotic $\mathrm{Cl}$ contains additional subunits (known as accessory subunits) that might have been acquired during evolution to enrich $\mathrm{Cl}$ with new functions and regulatory properties. Interestingly $\mathrm{Cl}$ has been lost in certain eukaryote lineages such as the one leading to the yeast $S$. cerevisiae. However, other fungi such as Yarrowia lipolytica and Neurospora crassa do contain $\mathrm{Cl}$ and have been used as simple models for its study [102,103].

Like Yarrowia and Neurospora, Dictyostelium possesses $\mathrm{Cl}$ and homologous genes encoding Complex I subunits can be recognized in the mitochondrial and nuclear genomes. Also like them, Dictyostelium has proteins similar to all core subunits and a subset of accessory proteins (Supplementary Table 5). Interestingly the Dictyostelium mitochondrial genome codes for three $\mathrm{Cl}$ subunits that are nuclear encoded in other organisms [104]. This is consistent with divergence of major eukaryotic lineages before the process of transferring mitochondrial genes to the nuclear genome had been completed.

Despite its complexity only few assembly factors have been described to be required for the correct assembly and stability of this huge multiprotein complex. Six have been described to be involved in human Cl deficiency: NDUFAF1, NDUFAF2, NDUFAF3 and NDUFAF4 [105-108], C8orf38 [109] and C20orf7 $[110,111]$. Others are required for $\mathrm{Cl}$ assembly but have not yet been implicated in human disease such as Ecsit [112], AlF [113], Indl [114,115] and MidA/C2orf56 [17]. Interestingly, two (C20orf7 and MidA) are putative methyltransferases suggesting that methylation might play a relevant role in $\mathrm{Cl}$ function.

MidA is a conserved protein that was described for the first time in Dictyostelium as a mitochondrial protein required for cellular bioenergetics since null mutants have reduced ATP levels and are compromised in growth and development [63]. Bioinformatics modeling and site directed mutagenesis suggested that MidA contains a methyltransferase domain required for its function. The protein's ability to complement the mutant phenotype is abolished by mutations in a critical glycine in the catalytic core that is predicted to interact with the methyl donor S-adenosyl methionine (SAM) [17]. Dictyostelium cells lacking MidA show a specific defect in Complex I activity and this role has also been confirmed in mammalian cells since the knock-down of human MidA in HEK293T cells results in an isolated defect in the activity and assembly of Complex I. Both Dictyostelium and human MidA interact with the $\mathrm{Cl}$ core subunit NDUFS2, but the consequences of this interaction and whether or not MidA is involved in Ndufs2 methylation remain 
open questions [17]. Only two protein methylation modifications have been detected in Complex I subunits. One of them is in the bovine NDUFB3 (B12) subunit that is methylated at conserved His residues but has no homologue in Dictyostelium. Interestingly, the other one is the human NDUFS2 subunit that harbors a methylated arginine, R323 [116].

MidA knockout in Dictyostelium causes a complex phenotype that shows similarities but also differences with that of other mitochondrially diseased strains (Table 1 and Figure 2). Defects in phototaxis and thermotaxis, a hallmark of Dictyostelium mitochondrial defects, are present in the MidA mutant and can be rescued by AMPK antisense inhibition, similarly to the other described mitochondrial mutants $[9,17]$. However, midA cells are also defective in phagocytosis and macropinocytosis, a phenotype that is not rescued by AMPK antisense inhibition and is not even present in the other described mitochondrial mutants. These results suggest that isolated defects in $\mathrm{Cl}$ might cause abnormal phagocytosis and pinocytosis. Alternatively, MidA might have additional functions beyond $\mathrm{Cl}$ that could affect these cellular activities. The study of other $\mathrm{Cl}$-specific mutants in Dictyostelium is necessary to distinguish between these possibilities and to clarify the complex cytopathology associated with $\mathrm{Cl}$ disease.

\section{Conclusion}

The Dictyostelium model for mitochondrial disease is proving to be a rich field for investigation. It is simple and tractable enough to yield consistent, reproducible phenotypes in clonally derived mutants, yet has a sufficiently complex life cycle with unicellular and multicellular motile forms to provide a rich lode of phenotypic readouts of varied intracellular signalling pathways. It has taught us that sublethal mitochondrial dysfunction at the cellular level produces phenotypic outcomes from disturbed intracellular signalling networks not merely ATP depletion, cumulative oxidative damage and cell death. The implication is that whole organism pathology may arise earlier and be more nuanced than is often supposed - an outcome of sublethal cellular dysfunction not simply the result of cell death. It is to be hoped that with this insight and greater understanding of the nature of the cellular disturbances produced by mitochondrial disease, new treatment strategies will present themselves for these currently untreatable disorders.

\section{Acknowledgements}

This work was supported by grants BMC2006-00394 and BMC2009-09050 to R.E. from the Spanish Ministerio de Ciencia e Innovación and to P.R.F. from the Thyne Reid Memorial Trusts and the Australian Research Council. 


\section{Tables.}

Table 1. Phenotypes associated with mitochondrial dysfunction in Dictyostelium.

\begin{tabular}{|c|c|c|c|c|c|c|c|c|c|c|c|}
\hline \multirow{2}{*}{$\begin{array}{l}\text { Method of } \\
\text { generating } \\
\text { mitochondrial } \\
\text { dysfunction }\end{array}$} & \multicolumn{11}{|l|}{ Phenotype } \\
\hline & $\begin{array}{l}\text { Growth on } \\
\text { bacteria }\end{array}$ & $\begin{array}{l}\text { Growth in } \\
\text { broth }\end{array}$ & Phagocytosis & Pinocytosis & Phototaxis & Thermotaxis & Morphogenesis & Aggregation & Chemotaxis & $\begin{array}{l}\text { Legionella } \\
\text { susceptibility }\end{array}$ & Reference \\
\hline \multicolumn{12}{|c|}{ Pharmacological, expected to affect respiration } \\
\hline $\begin{array}{l}\text { Ethidium bromide } \\
\text { inhibition of mtDNA } \\
\text { replication }\end{array}$ & & - & & & - & & - (Stalky) & - & & & 57 \\
\hline \multicolumn{12}{|c|}{ Genetic, expected to affect respiration } \\
\hline $\begin{array}{l}\text { Heteroplasmic } \\
\text { mitochondrial gene } \\
\text { disruption ( } r n l \text {, nad5, } \\
\text { cob, nad2, atp6, atp1, } \\
\text { cox3, ORF1740, } \\
\text { ORF796) }\end{array}$ & - & - & + & + & - & - & - (Stalky) & - & & - & $7,11,50$ \\
\hline $\begin{array}{l}\text { Heteroplasmic rps } 4 \\
\text { disruption }\end{array}$ & & $+/-$ & & & - & & & - & & & $\begin{array}{l}\text { 52, Fisher } \\
\text { (unpublished) }\end{array}$ \\
\hline $\begin{array}{l}\text { Chaperonin } 60 \\
\text { antisense inhibition }\end{array}$ & - & - & + & + & - & - & - (Stalky) & - & & - & $8,9,11$ \\
\hline \multicolumn{12}{|c|}{ Genetic, respiratory complex-specific defect in respiration } \\
\hline $\begin{array}{l}\text { MidA knockout } \\
\text { producing specific } \\
\text { Complex I deficiency }\end{array}$ & - & - & - & - & - & - & +/- (Stalky) & + & & & 63,17 \\
\hline
\end{tabular}




\begin{tabular}{|c|c|c|c|c|c|c|c|}
\hline $\begin{array}{l}\text { Nuclear } f_{S Z A}, f_{S Z B} \\
\text { disruption }\end{array}$ & $\begin{array}{l}+\left(\mathrm{fszA}^{\mathrm{A}}\right) \\
-\left(\mathrm{fszB} \mathrm{B}^{-}\right)\end{array}$ & $\begin{array}{l}+\left(\mathrm{fszA}^{\prime}\right) \\
-\left(\mathrm{fszB}{ }^{-}\right)\end{array}$ & & + & + & + & $\begin{array}{l}\text { 55, Fisher } \\
\text { (unpublished) }\end{array}$ \\
\hline $\begin{array}{l}\text { Nuclear cluA } \\
\text { disruption }\end{array}$ & $\begin{array}{l}\text { - (Defective } \\
\text { cytokinesis) }\end{array}$ & $\begin{array}{l}\text { - (Defective } \\
\text { cytokinesis) }\end{array}$ & & & + & + & 54 \\
\hline $\begin{array}{l}\text { Nuclear torA } \\
\text { disruption }\end{array}$ & - & & + & & & - & 53 \\
\hline $\begin{array}{l}\text { Nuclear Dd-TRAP1 } \\
\text { RNAi inhibition }\end{array}$ & & - & & & & - & 56 \\
\hline $\begin{array}{l}\text { Nuclear aoxA } \\
\text { disruption }\end{array}$ & & + & & & + & + & 64 \\
\hline
\end{tabular}

+ , Wild type phenotype.

-, Aberrant phenotype.

+/-, Mildly aberrant phenotype.

Shaded cells, Phenotype not reported 


\section{References:}

1. Wallace DC. Mitochondrial DNA Mutations in Disease and Aging. Environ Mol Mut 2010;51(5):440-50.

2. Francione L, Fisher PR. Cytopathological mechanisms in mitochondrial disease. Curr Chem Biol 2010;4:32-48.

3. Zeviani M, Carelli V. Mitochondrial disorders. Current Opinion in Neurology. 2007:20(5):564-71.

4. Bender A, Krishnan KJ, Morris CM, Taylor GA, Reeve AK, Perry RH, et al. High levels of mitochondrial DNA deletions in substantia nigra neurons in aging and Parkinson disease. Nat Genet 2006;38:515-7.

5. Coskun PE, Beal MF, Wallace DC. Alzheimer's brains harbor somatic mtDNA control-region mutations that suppress mitochondrial transcription and replication. Proc Natl Acad Sci USA 2004;101:10726-31.

6. Kraytsberg Y, Kudryavtseva E, McKee AC, Geula C, Kowall NW, Khrapko, K. Mitochondrial DNA deletions are abundant and cause functional impairment in aged human substantia nigra neurons. Nat Genet 2006;38:518-520.

7. Wilczynska Z, Barth C, Fisher PR. Mitochondrial mutations impair signal transduction in Dictyostelium discoideum slugs. Biochem Biophys Res Comm $1997 ; 234: 39-43$.

8. Kotsifas M, Barth C, Lay ST, de Lozanne A, Fisher PR. Chaperonin 60 and mitochondrial disease in Dictyostelium. J Muscle Res Cell Motil 2002;23:839-52.

9. Bokko PB, Francione L, Bandala-Sanchez E, Ahmed AU, Annesley SJ, Huang X, et al. Diverse cytopathologies in mitochondrial disease are caused by AMP-activated protein kinase signaling. Mol Biol Cell 2007;18:1874-86.

10. Annesley SJ, Fisher PR. Dictyostelium discoideum - a model for many reasons. Mol Cell Biochem 2009;329:73-91.

11. Francione L, Smith PK, Accari SL, Taylor PE, Bokko PB, Bozzaro S, et al. Legionella pneumophila multiplication is enhanced by chronic AMPK signalling in mitochondrially diseased Dictyostelium cells. Dis Model Mech 2009;2:479-89.

12. Ogawa S, Yoshino R, Angata K, Iwamoto M, Pi M, Kuroe K, et al. The mitochondrial DNA of Dictyostelium discoideum: Complete sequence, gene content and genome organisation. Mol Gen Genet 2000;263:514-19.

13. Barth C, Greferath U, Kotsifas M, Fisher PR. Polycistronic transcription and editing of the mitochondrial small subunit (SSU) ribosomal RNA in Dictyostelium discoideum. Curr Genet 1999;36:55-61. 
14. Barth C, Greferath U, Kotsifas M, Tanaka Y, Alexander S, Alexander H, et al. Transcript mapping and processing of mitochondrial RNA in Dictyostelium discoideum. Curr Genet 2001;39:355-64.

15. Le $\mathrm{P}$, Fisher PR, Barth C. Transcription of the Dictyostelium discoideum mitochondrial genome occurs from a single initiation site. RNA 2009;15:2321-30.

16. Ruderman NB, Xu XJ, Nelson L, Cacicedo JM, Saha AK, Lan F, et al. AMPK and SIRT1: a long-standing partnership? Am J Physiol Endocrinol Metab 2010;298(4):E751-E60.

17. Carilla-Latorre S, Gallardo ME, Annesley SJ, Calvo-Garrido J, Grana O, Accari SL, et al. MidA is a putative methyltransferase that is required for mitochondrial complex I function. J Cell Sci 2010;123:1674-83.

18. Wallace DC, Fan WW. Energetics, epigenetics, mitochondrial genetics. Mitochondrion 2010;10(1):12-31.

19. Leonard JV, Schapira, AHV. Mitochondrial respiratory chain disorders I: mitochondrial DNA defects. Lancet 2000;355:299-304.

20. Shutt TE, Shadel GS. A Compendium of Human Mitochondrial Gene Expression Machinery With Links to Disease. Environ Mol Mutag 2010;51(5):360-79.

21. Barth C, Le P, Fisher PR. Mitochondrial biology and disease in Dictyostelium. Intern Rev Cytol 2007;263:207-52.

22. Bolender N, Sickmann A, Wagner R, Meisinger C, Pfanner N. Multiple pathways for sorting mitochondrial precursor proteins. EMBO Rep 2008;9:42-9.

23. Bonawitz ND, Clayton DA, Shadel GS. Initiation and beyond: multiple functions of the human mitochondrial transcription machinery. Mol Cell 2006;24:813-25.

24. Koehler CM, Leuenberger, D, Merchant S, Renold A, Junne T, Schatz G. Human Deafness Dystonia Syndrome is a mitochondrial disease. Proc Natl Acad Sci USA $1999 ; 96,2141-46$.

25. Hood, D. A. \& Joseph, A. M. Mitochondrial assembly: protein import. Proc Nutr Soc $2004 ; 63: 293-300$.

26. Baker MJ, Frazier AE, Gulbis JM, Ryan MT. Mitochondrial protein-import machinery: correlating structure with function. TRENDS Cell Biol 2007;17:456-64.

27. Macasev D, Whelan J, Newbigin E, Silva-Filho MC, Mulhern TD, Lithgow T. Tom22', an 8-kDa trans-site receptor in plants and protozoans, is a conserved feature of the TOM complex that appeared early in the evolution of eukaryotes. Mol Biol Evol 2004;21:1557-64.

28. Ahmed AU, Beech PL, Lay ST, Gilson PR, Fisher PR. Import-associated translational inhibition-novel in vivo evidence for cotranslational protein import into Dictyostelium mitochondria. Euk Cell 2006;5:1314-27.

29. Ahmed AU, Fisher PR. Import of nuclear-encoded mitochondrial proteins: a cotranslational perspective. Int Rev Cell Mol Biol 2009;273:49-68. 
31. Holt IJ, Harding AE, Petty RK, Morgan-Hughes JA. A new mitochondrial disease associated with mitochondrial DNA heteroplasmy. Am J Hum Genet 1990;46:428-33.

30. Lithgow T. Targeting of proteins to mitochondria. FEBS Lett 2000;476:22-6.

32. Tatuch Y, Christodoulou J, Feigenbaum A, Clarke JT, Wherret J, Smith C, et al. Heteroplasmic mtDNA mutation ( $T-G)$ at 8993 can cause Leigh disease when the percentage of abnormal DNA is high. Am J Hum Genet 1992;50:852-8.

33. Kraytsberg Y, Kudryavtseva E, McKee AC, Geula C, Kowall NW, Khrapko K. Mitochondrial DNA deletions are abundant and cause functional impairment in aged human substantia nigra neurons. Nat Genet 2006;38:518-520.

34. Rossignol R, Faustin B, Rocher C, Malgat M, Mazat J, Letellier T. Mitochondrial threshold effects. Biochem J 2003;370:751-62.

35. Eichinger L, Pachebat JA, Glöckner G, Rajandream M-A, Sucgang R, Berriman M, et al. The genome of the social amoeba Dictyostelium discoideum. Nature 2005;435:43-57.

36. Williams RS, Boeckeler K, Gräf R, Müller-Taubenberger A, Li Z, Isberg RR, et al. Towards a molecular understanding of human diseases using Dictyostelium discoideum. Trends Mol Med 2006;12:415-24.

37. Jin $T, X u X$, Hereld D. Chemotaxis, chemokine receptors and human disease. Cytokine;2008;44:1-8.

38. Liao XH, Majithia A, Huang X, Kimmel AR. Growth control via TOR kinase signaling, an intracellular sensor of amino acid and energy availability, with crosstalk potential to proline metabolism. Amino Acids 2008;35:761-70.

39. Swaney KF, Huang $\mathrm{CH}$, Devreotes PN. Eukaryotic chemotaxis: a network of signaling pathways controls motility, directional sensing, and polarity. Annu Rev Biophys 2010;39:265-89.

40. Cosson P, Soldati T. Eat, kill or die: when amoeba meets bacteria. Curr Opin Microbiol 2008;11:271-6.

41. Fisher PR. Genetics of phototaxis in a model eukaryote, Dictyostelium discoideum. Bioessays 1997;19:397-408.

42. Fisher PR. Genetic analysis of phototaxis in Dictyostelium. In: Hader D-P, Lebert $\checkmark$ editors. Photomovement. ESP Comprehensive Series in Photosciences, Amsterdam: Elsevier Science Ltd; 2001, Vol 1, pp. 519-59.

43. Williams JG . Transcriptional regulation of Dictyostelium pattern formation. EMBO Rep 2006;7:694-8.

44.Calvo-Garrido J, Carilla-Latorre S, Kubohara Y, Santos-Rodrigo N, Mesquita A, Soldati T et al. Autophagy in Dictyostelium: genes and pathways, cell death and infection. Autophagy 2010;16:686-701.

45. Weijer CJ. Collective cell migration in development. J Cell Sci 2009;122:3215-23. 
46. Dormann D, Weijer CJ. Chemotactic cell movement during Dictyostelium development and gastrulation. Curr Opin Genet Dev 2006;16:367-73

47. Clarke M. Recent insights into host-pathogen interactions from Dictyostelium. Cell Microbiol 2010;12:283-91.

48. Albert-Weissenberger C, Cazalet C, Buchrieser, C. Legionella pneumophila - a human pathogen that co-evolved with fresh water protozoa. Cell Mol Life Sci 2007;64:432-48.

49. Hilbi H, Weber SS, Ragaz C, Nyfeler Y, Urwyler S. Environmental predators as models for bacterial pathogenesis. Environ Microbiol 2007;9:563-75.

50. Francione L (2008) Mitochondrial disease in Dictyostelium discoideum. PhD Thesis. La Trobe University.

51. Morita T, Yamaguchi $H$, Amagai A, Maeda $Y$ (2005). Involvement of the TRAP-1 homologue, Dd-TRAP1, in spore differentiation during Dictyostelium development. Exp Cell Res 2005;303:425-31.

52. Inazu Y, Chae SC, Maeda Y. Transient expression of a mitochondrial gene cluster including rps4 is essential for the phase-shift of Dictyostelium cells from growth to differentiation. Dev Genet 1999; 25:339-52.

53. van Es S, Wessels D, Soll DR, Borleis J, Devreotes PN. Tortoise, a novel mitochondrial protein, is required for directional responses of Dictyostelium in chemotactic gradients. J Cell Biol 2001;152:621-32.

54. Zhu Q, Hulen D, Liu T, Clarke M. The cluA- mutant of Dictyostelium identifies a novel class of proteins required for dispersion of mitochondria. Proc Natl Acad Sci USA 1997;94:7308-13.

55. Gilson PR, Yu X-C, Hereld D, Barth C, Savage A, Kiefel BR, et al. Two Dictyostelium orthologs of the prokaryotic cell division protein FtsZ localize to mitochondria and are required for the maintenance of normal mitochondrial morphology. Eukaryot Cell 2003;2:1315-26.

56. Morita T, Amagai A, Maeda Y. Translocation of the Dictyostelium TRAP1 homologue to mitochondria induces a novel prestarvation response. J Cell Sci 2004;117:5759-70.

57. Chida J, Yamaguchi $H$, Amagai A, Maeda $Y$. The necessity of mitochondrial genome DNA for normal development of Dictyostelium cells. J Cell Sci 2004; 117:3141-52.

58. Agsteribbe E, Huckriede A, Veenhuis M, Ruiters MHJ, Niezen-Koning KE, Skjeidal $\mathrm{OH}$, et al. A fatal, systemic mitochondrial disease with decreased mitochondrial enzyme activities, abnormal ultrastructure of the mitochondria and deficiency of heat shock protein 60. Biochem Biophys Res Comm 1993;193:146-54.

59. Huckriede A, Agsteribbe E. Decreased synthesis and inefficient mitochondiral import of Hsp60 in a patient with a mitochondrial encephalomyopathy. Biochim Biophy Acta 1994;1227:200-6. 
60. Briones P, Vilaseca MA, Ribes A, Vernet A, Lluch M, Cusi V, et al. A new case of multiple mitochondrial enzyme deficiencies with decreased amount of heat shock protein 60. J Inherit Metab Dis 1997;20:569-77.

61. Barth C, Fraser DJ, Fisher PR. Co-insertional replication is responsible for tandem multiple formation during plasmid integration into the Dictyostelium genome. Plasmid 1998;39:141-53.

62. Barth C, Fraser DJ, Fisher PR. A rapid, small scale method for characterization of plasmid insertions in the Dictyostelium genome. Nucl Acids Res 1998;26: 3317-8.

63. Torija P, Vicente JJ, Rodrigues TB, Robles A, Cerdan S, Sastre L, et al. Functional genomics in Dictyostelium: MidA, a new conserved protein, is required for mitochondrial function and development. J Cell Sci 2006;119(Pt 6):1154-64.

64. Kimura K, Kuwayama H, Amagai A, Maeda S. Developmental significance of cyanide-resistant respiration under stressed conditions: Experiments in Dictyostelium cells. Develop Growth Differ 2010;52:645-56.

65. Wilkins A, Khosla M, Fraser DJ, Spiegelman GB, Fisher PR, Weeks G, et al. Dictyostelium RasD is required for normal phototaxis, but not differentiation. Genes Devel 2000;14:1407-13.

66. Wilkins A, Szafranski K, Fraser DJ, Bakthavatsalam D, Muller R, Fisher PR, et al. The Dictyostelium genome encodes numerous RasGEFs with multiple biological roles. BMC Genome Biol 2005;6:R68.

67. Bandala-Sanchez E, Annesley SJ, Fisher PR. A phototaxis signalling complex in Dictyostelium discoideum. Eur J Cell Biol 2006;85:1099-106.

68. Stocker S, Hiery M, Marriot G. Phototactic migration of Dictyostelium cells is linked to a new type of gelsolin-related protein. Mol Biol Cell 1999;10:161-78.

69. Gloss A, Rivero F, Khaire N, Muler R, Loomis WF, Schleicher M, et al. Villidin, a novel WD-repeat and villin-related protein from Dictyostelium, is associated with membranes and the cytoskeleton. Mol Biol Cell 2003;14:2716-27.

70. Noegel AA, Blau-Wasser $R$, Sultana $H$, Israel $L$, Schleicher $M$, Patel $H$, et al. The cyclase-associated protein CAP as regulator of cell polarity and CAMP signalling in Dictyostelium. Mol Biol Cell 2004;15:934-45.

71. Fisher PR, Noegel AA, Fechheimer M, Rivero F, Prassler J, Gerisch G. Photosensory and thermosensory responses in Dictyostelium slugs are specifically impaired by absence of the F-actin cross-linking gelation factor (ABP-120). Current Biol 1997; 7:889-92.

72. Knuth M, Khaire N, Kuspa A, Lu SJ, Schleicher M, Noegel AA. A novel partner for Dictyostelium filamin is an a-helical developmentally regulated protein. J Cell Sci 2004;117:5013-22.

73. Annesley SJ, Bandala-Sanchez E, Ahmed AU, Fisher PR. Filamin repeat segments required for photosensory signalling in Dictyostelium discoideum. BMC Cell Biol 2007;8:48. 
74. Inoki K, Corradetti MN, Guan KL. Dysregulation of the TSC-mTOR pathway in human disease. Nat Genet 2005;37:19-24.

75. Gwinn DM, Shackelford DB, Egan DF, Mihaylova MM, Mery A, Vasquez DS, et al. AMPK phosphorylation of RapTOR mediates a metabolic checkpoint. Mol Cell 2008;30:214-26.

76. Choi JS, Park C, Jeong JW. AMP-activated protein kinase is activated in Parkinson's disease models mediated by 1-methyl-4-phenyl-1,2,3,6tetrahydropyridine. Biochem Biophys Res Comm 2010;39:1147-151.

77. Lopez-Lopez C, Dietrich MO, Metzger F, Loetscher H, Torres-Aleman I. Disturbed Cross Talk between Insulin-Like Growth Factor I and AMP-Activated Protein Kinase as a Possible Cause of Vascular Dysfunction in the Amyloid Precursor Protein/Presenilin 2 Mouse Model of Alzheimer's Disease. J Neurosci 2007;27:824-31.

78. Chou SY, Lee YC, Chen HM, Chiang MC, Lai HL, Chang HH, et al. CGS21680 attenuates symptoms of Huntington's disease in a transgenic mouse model. J Neurochem 2005;93(2):310-20.

79. Matsuyuma S, Maeda Y. Involvement of cyanide-resistant respiration in cell-type proportioning during Dictyostelium development. Dev Biol 1995;172:182-91.

80. Liesa M, Palacin M, Zorzano A. Mitochondrial dynamics in health and disease. Physiol Rev 2009;89:799-845.

81. Wienke DC, Knetsch MLW, Neuhais EM, Reedy MC, Manstein DJ. Disruption of a dynamin homologue affects endocytosis, organelle morphology and cytokinesis in Dictyostelium discoideum. Mol Biol Cell 1999;10:225-43.

82. Fields SD, Conrad MN, Clarke M. The S. cerevisiae CLU1 and D. discoideum cluA genes are functional homologues that influence mitochondrial morphology and distribution. J Cell Sci 1998;111:1717-27.

83. Cechetto JD, Gupta RS. Immunoelectron microscopy provides evidence that tumor necrosis factor receptor-associated protein 1 (TRAP1) is a mitochondrial protein which also localises at specific extramitochondrial sites. Exp Cell Res 2000;260:30-9.

84. Morita T, Amagai A, Maeda Y. Unique behavior of a Dictyostelium homologue of TRAP-1, coupling with differentiation of $D$. discoideum cells. Exp Cell Res 2002;208:45-54.

85. Yamaguchi H, Morita T, Amagai A, Maeda Y. Changes in spatial and temporal localization of Dictyostelium homologues of TRAP1 and GRP94 revealed by immnunoelectron microscopy. Exp Cell Res 2005;303:415-24.

86. Jarmuszkiewicz W, Behrendt M, Navet R, Sluse FE. Uncoupling protein and alternative oxidase of Dictyostelium discoideum: occurrence, properties and protein expression during vegetative life and starvation-induced early development. FEBS Lett 2002;532:459-64. 
87. Hardie DG, Sakamoto K. AMPK: A key sensor of fuel and energy status in skeletal muscle. Physiology 2006;21:48-60.

88. Hong SP, Leiper FC, Woods A, Carling D, Carlson M. Activation of yeast Snf1 and mammalian AMP-activated protein kinase by upstream kinases. Proc Natl Acad Sci USA 2003;100:8839-43.

89. Woods A, Johnstone SR, Dickerson K, Leiper FC, Fryer LG, Neumann D, et al. LKB1 is the upstream kinase in the AMP-activated protein kinase cascade. Curr Biol 2003;13:2004-8.

90. Hong SP, Momcilovic M, Carlson M. Function of mammalian LKB1 and $\mathrm{Ca}^{2+} /$ calmodulin-dependent protein kinase kinase a as Snf1-activating kinases in yeast. J Biol Chem 2005;280:21804-9.

91. Hurley RL, Anderson KA, Franzone JM, Kemp BE, Means AR, Witters LA. The $\mathrm{Ca}^{2+} /$ calmodulin-dependent protein kinase kinases are AMP-activated protein kinase kinases. J Biol Chem 2005;280:29060-6.

92. Momcilovic M, Hong S-P, Carlson M. Mammalian TAK1 activates Snf1 protein kinase in yeast and phosphorylates AMP-activated protein kinase in vitro. J Biol Chem $2006 ; 281: 25336-43$.

93. Kurth-Kraczek EJ, Hirshman MF, Goodyear LJ, Winder WW. 5' AMP-activated protein kinase activation causes GLUT4 trasnlocation in skeletal muscle. Diabetes 1999;48:1667-71.

94. Inoki K, Zhu T, Guan KL. TSC2 mediates cellular energy response to control cell growth and survival. Cell 2003;115:577-90.

95. Horman S, Browne G, Krause U, Patel J, Vertommen D, Bertrand L, et al. Activation of AMP-Activated Protein Kinase Leads to the Phosphorylation of Elongation Factor 2 and an Inhibition of Protein Synthesis. Curr Biol 2002;12:141923.

96. Bergeron R, Ren JM, Cadman KS, Moore IK, Perret P, Pypaert M, et al. Chronic activation of AMP kinase results in NRF-1 activation and mitochondrial biogenesis. Am J Physiol Endocrinol Metab 2001;281:E1340-E6.

97. Zong H, Ren JM, Young LH, Pypaert M, Mu J, Birnbaum MJ, et al. AMP kinase is required for mitochondrial biogenesis in skeletal muscle in response to chronic energy deprivation. Proc Natl Acad Sci USA 2002;99:15983-7.

98. Koopman WJ, Nijtmans LG, Dieteren CE, Roestenberg P, Valsecchi F, Smeitink JA, Willems PH. Mammalian mitochondrial complex I: biogenesis, regulation, and reactive oxygen species generation. Antioxid Redox Signal 2010;12(12):1431-70

99. Janssen RJ, Nijtmans LG, van den Heuvel LP, Smeitink JA. Mitochondrial complex I: structure, function and pathology. J Inherit Metab Dis 2006;29(4):499-515

100. Lazarou M, Thorburn DR, Ryan MT, McKenzie M. Assembly of mitochondrial complex I and defects in disease. Biochim Biophys Acta 2009;1793:78-88. 
101. Sharma LK, Lu J, Bai Y. Mitochondrial respiratory complex I: structure, function and implication in human diseases. Curr Med Chem 2009;16(10):1266-77.

102. Brandt U, Abdrakhmanova A, Zickermann V, Galkin A, Drose S, Zwicker K. et al. Structure-function relationships in mitochondrial complex I of the strictly aerobic yeast Yarrowia lipolytica. Biochem Soc Trans 2005;33(Pt 4):840-4.

103. Videira A, Duarte M. From NADH to ubiquinone in Neurospora mitochondria. Biochim Biophys Acta 2002;1555(1-3):187-91.

104. Cole RA, Slade MB, Williams KL. Dictyostelium discoideum mitochondrial DNA encodes a NADH:ubiquinone oxidoreductase subunit which is nuclear encoded in other eukaryotes. J Mol Evol 1995;40:616-21

105. Hoefs SJ, Dieteren CE, Rodenburg RJ, Naess K, Bruhn H, Wibom R, et al. Baculovirus complementation restores a novel NDUFAF2 mutation causing complex I deficiency. Hum Mutat 2009;30(7):E728-36.

106. Ogilvie I, Kennaway NG, Shoubridge EA. A molecular chaperone for mitochondrial complex I assembly is mutated in a progressive encephalopathy. J Clin Invest 2005;115(10):2784-92.

107. Saada A, Vogel RO, Hoefs SJ, van den Brand MA, Wessels $\mathrm{HJ}$, Willems $\mathrm{PH}$, et al. Mutations in NDUFAF3 (C3ORF60), encoding an NDUFAF4 (C6ORF66)-interacting complex I assembly protein, cause fatal neonatal mitochondrial disease. Am J Hum Genet 2009;84(6):718-27.

108. Vogel RO, Janssen RJ, Ugalde C, Grovenstein M, Huijbens RJ, Visch HJ, et al. Human mitochondrial complex I assembly is mediated by NDUFAF1. FEBS 2005;272(20):5317-26.

109. Pagliarini DJ, Calvo SE, Chang B, Sheth SA, Vafai SB, Ong SE, et al. A mitochondrial protein compendium elucidates Compex I disease biology. Cell 2008;134:112-23.

110. Gerards $M$, Sluiter W, van den Bosch BJC, de Wit LEA, Calis CMH, Frentzen $M$, et al. Defective complex I assembly due to C20orf7 mutations as a new cause of Leigh syndrome. J Med Genet 2010;47:507-12.

111. Sugiana C, Pagliarini DJ, McKenzie M, Kirby DM, Salemi R, Abu-Amero KK, et al. Mutation of C20orf7 disrupts complex I assembly and causes lethal neonatal mitochondrial disease. Am J Hum Genet 2008;83(4):468-78.

112. Vogel RO, Janssen RJ, van den Brand MA, Dieteren CE, Verkaart S, Koopman WJ, et al. Cytosolic signaling protein Ecsit also localizes to mitochondria where it interacts with chaperone NDUFAF1 and functions in complex I assembly. Genes Dev 2007;21(5):615-24.

113. Vahsen N, Cande C, Briere J], Benit P, Joza N, Larochette N, et al. AIF deficiency compromises oxidative phosphorylation. EMBO J 2004;23(23):4679-89. 
114. Bych K, Kerscher S, Netz DJ, Pierik AJ, Zwicker K, Huynen MA, et al. The ironsulphur protein Ind1 is required for effective complex I assembly. EMBO J $2008 ; 27(12): 1736-46$

115. Sheftel AD, Stehling O, Pierik AJ, Netz DJ, Kerscher S, Elsasser HP, et al. Human ind1, an iron-sulfur cluster assembly factor for respiratory complex I. Mol Cell Biol 2009;29(22):6059-73

116. Carroll J, Fearnley IM, Shannon RJ, Hirst J, Walker JE. Analysis of the subunit composition of complex I from bovine heart mitochondria. Mol Cell Proteomics 2003;2(2):117-26. 


\section{Figure legends.}

Figure 1. Organisation, transcription and transcript processing of the Dictyostelium mitochondrial genome.

The Dictyostelium mitochondrial genes as well as introns and noncoding regions are shown. The genome is transcribed from a single start site as indicated by "Transcription start" [15]. The primary transcript may be rapidly and cotranscriptionally processed into smaller mature transcripts. Only the secondary transcripts A (3.1 kb), B (4.6 kb), C (5.6 kb), D (9.5 kb), E (6.0 kb), F (6.5 kb), G (3.7 kb), H $(8.7 \mathrm{~kb})$ and their smaller derivatives (tertiary transcripts) have been detected in northern hybridisation studies (Barth et al., 2001). From Fig. 1 of Barth et al. (2007) [21].

Figure 2. Cytopathological pathways in Dictyostelium mitochondrial disease.

Two pathways are shown. 1. Generalized mitochondrial respiratory dysfunction affecting multiple oxidative phosphorylation complexes compromises mitochondrial ATP generation, leading to chronic AMPK activation. The resulting dysregulation of intracellular signalling produces multiple cytopathological outcomes. Not shown is the homeostatic feedback by which AMPK stimulates mitochondrial biogenesis and ATP production. In mitochondrially diseased cells chronic AMPK activation can thereby maintain ATP at normal levels while at the same time causing chronic downstream cytopathologies. 2. In addition to AMPK-dependent pathways, a MidA-dependent dysfunction (possibly through Complex I-specific deficiency indicated by the question mark) impairs endocytic pathways (phagocytosis and macropinocytosis) in an AMPK-independent manner. Different, more limited cytopathologies may be caused by mutations that affect other aspects of mitochondrial biology without impairing ATP production. 


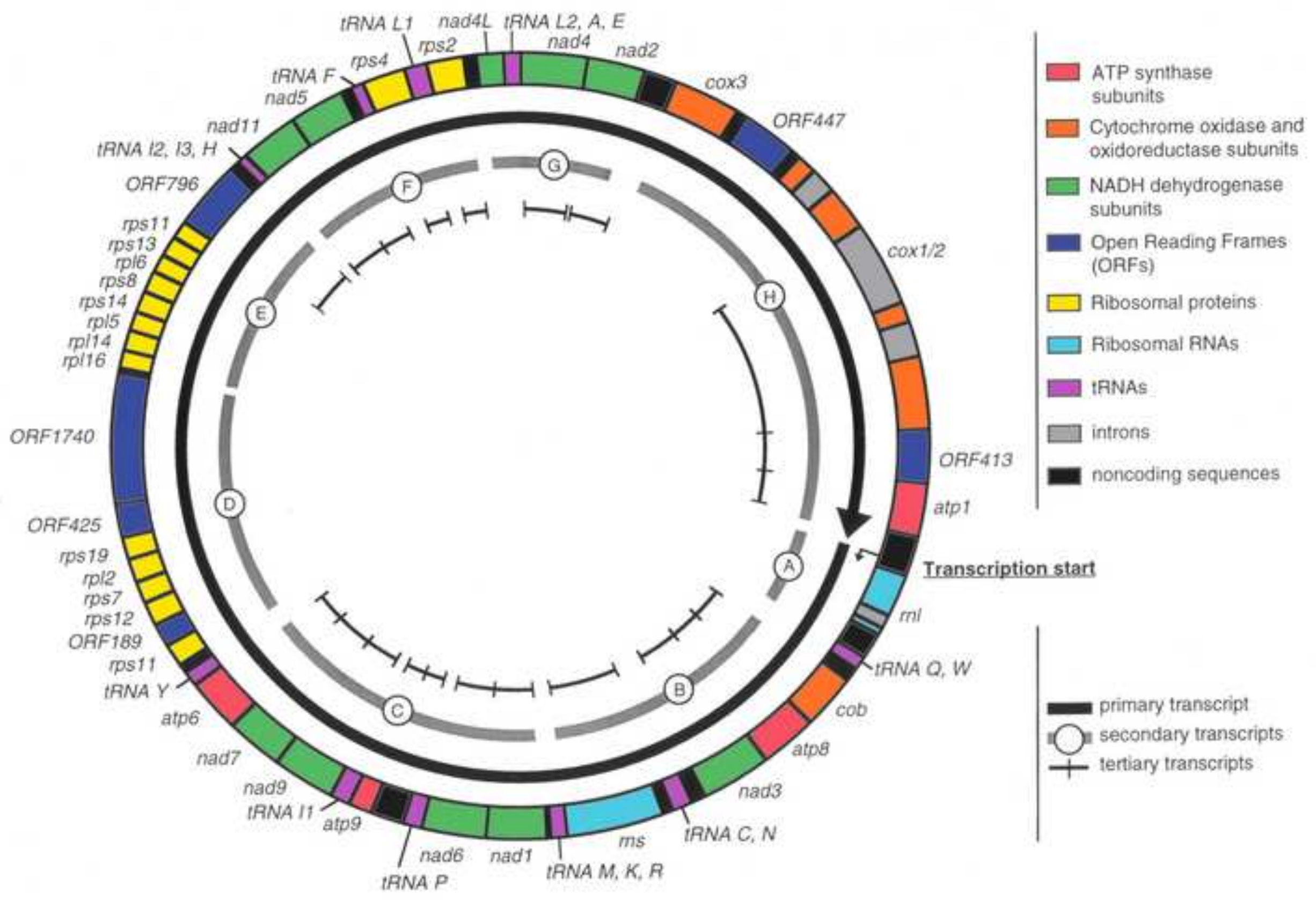

